\title{
O USO DA AVALIAÇÃO DO CICLO DE VIDA E DA ECOEFICIÊNCIA PARA AVALIAR ALTERNATIVAS DE VALORIZAÇÃO DE RESÍDUOS: UM ESTUDO EM EMPRESA TERMELETTRICA
}

\author{
USE OF LIFE CYCLE ASSESSMENT AND ECO-EFFICIENCY TO \\ EVALUATE ALTERNATIVE WASTE RECOVERY: A STUDY IN THE \\ THERMAL POWER PLANT
}

Data de submissão: 18-04-2016 Aceite: 13-11-2016

Mario Roberto dos Santos ${ }^{1}$

Cláudia Echevenguá Teixeira²

Cláudia Terezinha Kniess ${ }^{3}$ José Carlos Barbieri ${ }^{4}$

\section{RESUMO}

O objetivo deste artigo é apresentar um método para avaliar alternativas de valorização de resíduos usando de modo combinado a Avaliação do Ciclo de Vida (ACV) e medidas de ecoeficiência. Esse método foi aplicado às cinzas pesadas - resíduos provenientes da combustão de carvão mineral em usinas termelétricas - empregadas como matéria-prima alternativa à areia industrial utilizada na produção de materiais cerâmicos e também ao clínquer utilizado na produção de cimento Portland. A avaliação da ecoeficiência foi realizada conforme a norma NBR ISO 14045, que orienta que os impactos ambientais devem ser avaliados por meio da utilização da ACV. Os resultados mostraram que a ecoeficiência relativa da geração de cinzas pesadas para uso na indústria de revestimentos cerâmicos comparada com a geração de cinzas pela termelétrica dispostas nas lagoas passaria de $0,2 \%$ inferior para $0,97 \%$ superior. Já a ecoeficiência relativa da geração de cinzas pesadas para uso na indústria de cimento comparada com a geração de cinzas pela termelétrica dispostas nas lagoas passaria de $1 \%$ inferior para valores entre $2,4 \%$ e $11,5 \%$ superiores. A aplicação do método indicou, assim, que o uso das cinzas pesadas na produção de materiais cerâmicos ou na substituição do clínquer na produção de cimento Portland melhora a ecoeficiência das empresas termelétricas.

Palavras-chave: Avaliação do ciclo de vida (ACV), Carvão mineral, Cinzas pesadas, Ecoeficiência, Valorização de resíduos.

\footnotetext{
1 Possui graduação em Engenharia pela Escola de Engenharia Maua, IMT, mestrado em Ciências Contábeis pelo Centro Universitário FECAP, FECAP e doutorado em Administração pela Universidade Nove de Julho, UNINOVE. São Paulo. São Paulo. Brasil. E-mail: mario.rsantos@ terra.com

2 Possui graduação em Biologia pela Universidade de Caxias do Sul, UCS, mestrado em Engenharia Civil pela Universidade Estadual de Campinas, UNICAMP e doutorado em Engenharia Civil pela Université de Sherbrooke, USherbrooke, Canadá. São Paulo. São Paulo. Brasil. E-mail: ceteixeira10@gmail.com

3 Possui graduação em Bacharelado em Química pela Universidade Federal de Santa Catarina, UFSC, mestrado em Engenharia Química pela Universidade Federal de Santa Catarina, UFSC e doutorado em Ciência e Engenharia de Materiais pela Universidade Federal de Santa Catarina, UFSC. São Paulo. São Paulo. Brasil. E-mail: kniesscl@yahoo.com.br

4 Possui graduação em Administração Pública pela Fundação Getulio Vargas - SP, FGV, mestrado em Administração Pública pela Fundação Getulio Vargas - SP, FGV e doutorado em Administração Pública pela Fundação Getulio Vargas - SP, FGV. São Paulo. São Paulo. Brasil. E-mail: Jose.Barbieri@fgv.br
} 


\begin{abstract}
The aim of this paper is to present a method to assess alternatives for waste recovery using combined mode the Life Cycle Assessment (LCA) and eco-efficiency measures. This method applied in relation to the bottom ash - waste from mineral coal combustion in thermal power plants - as an alternative raw material in place of industrial sand in the production of ceramic materials and the replacement of clinker used in the Portland cement production. The evaluation of eco-efficiency was performed according to the NBR ISO 14045 standard that guides the environmental impacts should be evaluated using LCA. The results showed that the eco-efficiency on the generation of bottom ash for use in the ceramic industry compared with the generation of bottom ash and disposal of thermal power plant in surface impoundment would increase from $0.2 \%$ down to $0.97 \%$ higher. Eco-efficiency on the generation of bottom ash for use in the cement industry compared with the generation of bottom ash and disposal of thermal power plant in surface impoundment would increase from $1 \%$ down to values between $2.4 \%$ and $11.5 \%$ higher. The application of the method showed that the use of bottom ash as a raw material of ceramic productions or clinker substitution of Portland cement productions improves the eco-efficiency of thermal power plants.

Keywords: Life cycle assessment (LCA), Mineral coal, Bottom ash, Eco-efficiency, Waste recovery.
\end{abstract}

\title{
1 INTRODUÇÃO
}

A gestão ambiental no âmbito das empresas durante muito tempo resumiu-se praticamente ao controle da poluição para atender os padrões de emissão estabelecidos pelas políticas públicas ambientais e à correção para restaurar as características ambientais afetadas por descargas indevidas de poluentes no meio ambiente. Originam-se daí as duas abordagens típicas de gestão ambiental: a de controle da poluição ao final do processo (end-of-pipe) e a de remediação. No entanto, essas abordagens se mostraram insuficientes quando aplicadas de modo exclusivo tanto para as empresas quanto para o meio ambiente, o que levou a novas abordagens, tais como a da redução da geração de poluentes na fonte e a da valorização dos resíduos captados, requerendo, para isso, uma visão mais abrangente do processo produtivo. Assim, progressivamente a gestão ambiental passou de uma abordagem centrada no controle da poluição para uma abordagem centrada na prevenção ao longo do ciclo de vida do produto, isto é, ao longo da cadeia de suprimento.

Essas novas abordagens não eliminaram as atividades de controle; ao contrário, estas continuam necessárias, pois, como se sabe, não há processo de produção $100 \%$ eficiente, de modo que sempre será preciso controlar as emissões por melhor que sejam as práticas de prevenção. Além disso, há setores em que a geração de resíduos é elevada devido aos insumos utilizados e às suas características operacionais.

Quando a geração de resíduos é inevitável nos processos industriais e no ciclo de vida dos produtos, eles devem ser introduzidos em novos ciclos produtivos, evitando o descarte na natureza (Bautista-Lazo; Short, 2013). A crescente demanda de sistemas seguros de disposição final tem estimulado o estudo de alternativas tecnológicas e econômicas para que resíduos possam ser introduzidos como matéria-prima, carga e agregados em outros ciclos de produção. Dessa maneira, é possível diminuir os custos de tratamento e disposição final, além de oferecer matéria-prima secundária ao mercado (Teixeira, 2001; Tonini; Astrup, 2012).

Quando a origem e as características dos resíduos são conhecidas, sua incorporação no processo produtivo torna-se atraente ao desenvolvimento de novos produtos com propriedades de interesse tecnológico (Zanchet et al., 2007). Alguns aspectos referentes à introdução desses resíduos precisam ser estudados e compreendidos, para que a quantidade de resíduo adicionada seja tecnicamente viável e o produto final obtido possa ter um desempenho técnico semelhante ao obtido com a matéria-prima convencional (Chowdhury; Apul; Fry, 2010; Liang; Zhang, 2012; Merrild; Larsen; Christensen, 2012). 
O resíduo gerado pela combustão de carvão mineral nas termelétricas é um desses resíduos industriais de origem conhecida, de modo que o Brasil possui parte de sua matriz energética oriunda dessas usinas. Os principais impactos ambientais produzidos durante o beneficiamento do carvão mineral decorrem da disposição de resíduos sólidos e rejeitos sem valor comercial em áreas próximas ao local de sua utilização (Fungaro; Izidoro, 2006). As emissões para o ar originárias da combustão do carvão e seus impactos associados ao meio ambiente e à saúde humana têm sido historicamente a principal causa de preocupação com o uso de carvão para geração de energia elétrica (Babbitt; Lindner, 2005), uma vez que a cinza pesada originária do processo de combustão do carvão é depositada a céu aberto, em bacias de sedimentação, e carece de aplicações industriais (Kniess et al., 2011).

Asokan, Saxena e Asolekar (2005), Babbitt e Lindner (2005), Ramadoss e Sundararajan (2014), dentre outros autores, citam que a poluição ambiental, causada pelo carvão utilizado em usinas termelétricas e por seus resíduos, é vista como uma das principais fontes de poluição, afetando o ambiente no que concerne ao uso da terra, à saúde humana, ao ar, ao solo e à água e acarretando graves impactos ambientais. Portanto, a valorização e reutilização desses resíduos tornou-se uma questão importante nas últimas décadas (Menéndez et al., 2013). A valorização engloba diferentes oportunidades de agregar valor econômico aos resíduos, tais como a reciclagem, a compostagem, o aproveitamento energético de aterros e o coprocessamento de resíduos industriais, processos em que, segundo Paulli (1995 apud TEIXEIRA, 2001), os resíduos são considerados como fonte de matéria-prima para um novo ciclo de produção.

Nesse contexto, este artigo apresenta os resultados da aplicação de um método para avaliar alternativas de valorização de cinzas pesadas, provenientes da combustão de carvão mineral em usinas termelétricas, como matéria-prima alternativa à areia industrial empregada na produção de materiais cerâmicos e também ao clínquer usado na produção de cimento Portland. Para isso, este artigo está delineado da seguinte forma: após esta introdução, a seção dois apresenta uma breve revisão da literatura centrada na problemática da gestão de resíduos e, em especial, nos resíduos resultantes da queima de carvão mineral em termelétricas, abordando, ainda, a metodologia para calcular a ecoeficiência segundo a norma da Associação Brasileira de Normas Técnicas (ABNT) NBR ISO 14045; a seção três resume os procedimentos empregados para avaliar os impactos ambientais ao longo do ciclo de vida, bem como as ferramentas de análise; a seção quatro destina-se a apresentar e discutir os resultados da aplicação do método; e a última seção apresenta as considerações finais deste estudo.

\section{FUNDAMENTAÇÃO TEÓRICA}

Nesta seção, apresenta-se uma breve revisão da literatura sobre gestão de resíduos, cinzas pesadas de carvão mineral e ecoeficiência segundo a norma NBR ISO 14045.

\subsection{Gestão de resíduos}

A gestão de resíduos sólidos em seu modo tradicional é conhecida por contribuir significativamente para a criação de muitos problemas ambientais, tais como as alterações climáticas (emissões de gases de efeito estufa nos aterros), destruição do ozônio estratosférico (emissões de halocarbonos dos sistemas de refrigeração descartados), danos à saúde humana (exposição a produtos químicos e partículas durante o recolhimento e o tratamento de resíduos), danos ambientais (emissões de metais pesados para a atmosfera, o solo e a água) e esgotamento dos recursos (devido a sistemas inexistentes de reciclagem ou ineficientes para determinados minerais ou metais) (LAURENT et al., 2014a). 
Essa visão da gestão de resíduos, contudo, vem mudando, pois tal atividade está deixando de ser considerada somente uma atividade primária, focada no controle do fluxo de tratamento e da disposição final de resíduos gerados pela sociedade, para ser vista como uma atividade que pode contribuir para o fornecimento de energia e para a recuperação de recursos secundários (ASTRUP et al., 2015). Segundo Laurent et al. (2014b), os resíduos estão progressivamente deixando de ser vistos como uma fonte de poluição, para serem percebidos como uma fonte de recursos.

Nesse cenário, ganhos ambientais podem ser obtidos por meio de diferentes processos de gestão de resíduos, dentre os quais podem ser citados: incineração com reaproveitamento de energia; materiais reciclados utilizados como matérias-primas alternativas; tratamento biológico para produção de fertilizantes e combustíveis; incineração e, consequentemente, produção de material para a construção civil (EKVALL et al., 2007). Nas empresas, uma das metas da gestão de resíduos consiste na redução dos impactos das atividades industriais. Essas práticas podem, por exemplo, substituir materiais não biodegradáveis por biodegradáveis, reciclar produtos e reduzir consumo de energia e emissões atmosféricas. Quando tais práticas de redução de impactos ambientais têm como consequência o crescimento dos lucros, elas são aplicadas nas empresas como um todo (Hodge; Ochsendorf; Fernández, 2010). A esse respeito, Tian e Chen (2014) explicitam que as empresas têm aplicado o princípio dos 3R's (reduzir, reutilizar e reciclar) para a valorização de seus produtos, pois a reciclagem de recursos não é benéfica apenas para o meio ambiente, mas também para a inovação tecnológica.

Com o objetivo de minimizar o descarte de substâncias na natureza e de reduzir o consumo de recursos naturais, são gerados esforços no sentido de reintegrar os resíduos nos processos produtivos originais, seja por reciclagem, reuso ou recuperação, e assim possibilitar uma redução dos passivos ambientais (SANTOS; TEIXEIRA; KNIESS, 2014). Um dos resíduos que podem ser usados em outros processos industriais é a cinza pesada proveniente da combustão de carvão mineral.

\subsection{Cinzas pesadas de carvão mineral}

A combustão de carvão para a produção de energia elétrica em usinas termelétricas gera resíduos, isto é, cinzas pesadas e leves ou volantes. Babbitt e Lindner (2005) alertam que não é só na fase de combustão do carvão mineral que ocorrem problemas ambientais, mas durante todo o seu ciclo de vida, ou seja, durante a mineração, a lavagem e o transporte. Ruiz et al. (2014) relatam, por exemplo, problemas de conflitos socioambientais em decorrência da exploração de carvão, em consequência do afundamento do solo (subsidência) que ocorreu no município de Criciúma, estado de Santa Catarina, e em Illinois, nos Estados Unidos da América (EUA), relativos às minas subterrâneas antigas de carvão mineral. A proporção de cinzas geradas pela combustão do carvão mineral, contudo, não é constante em todas as usinas termelétricas, pois depende da configuração e operação da caldeira (Babbitt; Lindner, 2005) e da origem e das características do carvão mineral utilizado (Kniess, 2005).

A reciclagem de resíduos sólidos, por meio de sua incorporação em formulações cerâmicas para produção de cerâmicas tradicionais, tais como tijolos, telhas, revestimentos cerâmicos e porcelanas, tem atraído a atenção dos pesquisadores, em razão da possibilidade de aproveitamento de grandes quantidades de resíduos e dos resultados técnicos apresentados na literatura (Campos et al., 2007). Dentre esses resíduos, encontram-se as cinzas de carvão mineral, pois as suas características físicas, químicas e mineralógicas são compatíveis com várias matérias-primas utilizadas nas indústrias cerâmicas de revestimento, o que indica uma possibilidade de substituição parcial ou integral dessas matérias-primas pelo resíduo das cinzas de carvão mineral (KNIESS, 2005). 
Outro potencial uso para as cinzas pesadas é na indústria de cimento Portland (PILAR et al., 2016; VAN Oss; Padovani, 2003). Segundo o Sindicato Nacional da Indústria do Cimento (SNIC, 2015), a produção de cimento passa pelas seguintes fases de processamento: extração da matéria-prima; britagem; moagem da mistura crua; homogeneização da mistura crua; calcinação (clínquer); moagem do clínquer; e despacho do cimento.

\subsection{Ecoeficiência}

Ecoeficiência refere-se à combinação de duas dimensões da sustentabilidade, a econômica e a ambiental. Segundo Barbieri (2007, p. 138), “[...] a ecoeficiência baseia-se na ideia de que a redução de materiais e energia por unidade de produtos ou serviços aumenta a competitividade da empresa, ao mesmo tempo em que reduz as pressões sobre o meio ambiente, seja como fonte de recurso, seja como depósito de resíduos". Já para Glavic e Lukman (2007), trata-se da utilização mais eficiente de materiais e energia, a fim de obter mais rentabilidade e criar valor adicionado para um produto. Segundo a norma NBR ISO 14045 (2014, p. 2), ecoeficiência é o “[...] aspecto da sustentabilidade que relaciona o desempenho ambiental de um sistema de produto ao valor do sistema de produto".

Em termos gerais, a ecoeficiência é uma filosofia de gestão empresarial que incentiva a empresa a procurar melhorias ambientais que resultem em benefícios econômicos (Bréchet; Li, 2013). Segundo Knight e Jenkis (2009), utilizar o enfoque de ecoeficiência pode acarretar redução dos impactos de um processo produtivo industrial sobre o meio ambiente. Nesse sentido, "[...] a avaliação de ecoeficiência é uma ferramenta quantitativa de gestão que permite o estudo de impactos ambientais do ciclo de vida de um sistema de produto em conjunto com o valor do sistema de produto para uma parte interessada" (ABNT, 2014, p. 7).

Uma metodologia de avaliação de ecoeficiência foi criada no início da década de 1990 pelo World Business Council for Sustainable Development (WBCSD), em que a ecoeficiência é entendida como o quociente da divisão do valor do produto (medido em receita de vendas, quantidade de produção, preço do produto etc.) pela influência ambiental (medida em consumo de energia, de material, quantidade de poluentes emitidos etc.). Esse entendimento foi acatado pela norma ISO 14045 (ISO 2012), cujo texto foi adotado e traduzido pela ABNT em 2014, tornando-se a NBR ISO 14045: Gestão ambiental - Avaliação da ecoeficiência de sistemas de produto - Princípios, requisitos e orientações (ABNT, 2014). Segundo essa norma, a ecoeficiência é calculada pela fórmula:

$$
\text { Ecoeficiência }=\frac{\text { Valor do produto }}{\text { Impacto ambiental }}(\text { Equação } 1)
$$

A norma NBR ISO 14045 orienta também que, para a comparação da ecoeficiência entre produtos, seja usada a fórmula:

$$
\text { Fator }=\frac{\text { Ecoeficiência do produto avaliado }}{\text { Ecoeficiência do produto base }}(\text { Equação 2) }
$$

Neste estudo, a avaliação de ecoeficiência foi aplicada no processo de reutilização de cinzas pesadas, provenientes da combustão de carvão mineral, para a geração de energia elétrica em uma usina termelétrica. 


\section{MÉTODO}

A avaliação das alternativas de valorização das cinzas pesadas produzidas pela queima de carvão mineral foi realizada em uma empresa termelétrica, que é uma das maiores usinas geradoras de energia termelétrica por meio de combustão de carvão mineral do país e, portanto, um caso representativo no setor de geração de energia elétrica por combustão de carvão. Para isso, foram realizados os seguintes procedimentos metodológicos: (i) levantamento dos dados por meio de questionários e entrevistas em visitas à empresa em duas etapas; (ii) levantamento de dados secundários em teses, artigos de periódicos e base de dados do software SimaPro referentes aos inventários para a elaboração da Avaliação do Ciclo de Vida (ACV); e (iii) avaliação dos dados.

Um levantamento exploratório quantitativo foi realizado para dimensionar as emissões de poluentes na atmosfera, na água e no solo em termos numéricos relativos ao processo produtivo de energia elétrica e seus resíduos, com o foco nas cinzas pesadas. Foram dimensionadas, também, todas as emissões de poluentes na atmosfera, na água e no solo em termos numéricos relativos ao processo produtivo de areia para revestimentos cerâmicos e de clínquer para a indústria de cimento Portland.

A avaliação da ecoeficiência dos processos de geração de cinzas de carvão mineral, de produção e extração de areia para a indústria de revestimentos cerâmicos e de produção de clínquer para a indústria de cimento Portland foi realizada e comparada com o objetivo de mostrar que as cinzas pesadas podem ser utilizadas como matéria-prima alternativa tanto na produção de revestimentos cerâmicos quanto na produção de cimento Portland. Essa avaliação foi realizada seguindo a norma NBR ISO 14045 (2014), que orienta que os impactos ambientais sejam avaliados com a utilização da ACV, conduzida em conformidade com as normas NBR ISO 14040 (ABNT, 2009a) e NBR ISO 14044 (ABNT, 2009b).

\subsection{Objetivo e escopo da ACV}

A ACV avaliada teve como objetivos permitir a realização de: (i) um inventário das matérias-primas necessárias à produção dos resíduos gerados pela combustão de carvão (isto é, das cinzas pesadas de carvão mineral) e das emissões de poluentes associadas à sua produção; (ii) um inventário das matérias-primas necessárias à produção de areia destinada à produção de revestimentos cerâmicos e das emissões de poluentes associadas à sua produção; e (iii) um inventário das matérias-primas necessárias à produção dos resíduos gerados na produção do clínquer utilizado na fabricação de cimento Portland e das emissões de poluentes associadas à sua produção. Para a realização deste estudo, considerou-se como premissa que a substituição tanto da areia industrial quanto do clínquer por cinzas pesadas não interferirá nas propriedades dos revestimentos cerâmicos e do cimento Portland durante toda a sua vida útil, isto é, desde a sua fabricação até a sua disposição final.

O escopo da ACV incluiu:

a. produção das cinzas pesadas - mineração e preparação do carvão, transporte do carvão para a termelétrica, combustão do carvão, geração das cinzas pesadas, arraste hidráulico das cinzas para as lagoas e disposição nas lagoas da termelétrica;

b. produção das cinzas pesadas para substituição de areia industrial - mineração e preparação do carvão, transporte do carvão para a termelétrica, combustão do carvão, geração das cinzas pesadas, arraste hidráulico das cinzas para as lagoas, retirada da 
lagoa, secagem e transporte das cinzas até a indústria de revestimentos cerâmicos;

c. produção das cinzas pesadas para substituição de clínquer - mineração e preparação do carvão, transporte do carvão para a termelétrica, combustão do carvão, geração das cinzas pesadas, arraste hidráulico das cinzas para as lagoas, retirada da lagoa, secagem e transporte das cinzas até a indústria de cimentos Portland;

d. produção da areia - extração, produção e transporte até a indústria de produção de revestimentos cerâmicos;

e. produção de clínquer - extração das matérias-primas, transporte até a indústria de cimento Portland e produção do clínquer.

\subsection{Limites do sistema}

Os sistemas aqui considerados representam um processo típico de geração de energia por meio de carvão pulverizado e consequente produção de cinzas pesadas, produção de cerâmica e produção de cimento Portland. Os limites de cada estágio são apresentados nas Figuras 1 , 2 e 3 . O sistema inclui o transporte das cinzas pesadas desde a usina geradora até o seu local de uso, a indústria de produção de revestimentos cerâmicos e uma empresa de produção de cimento. Aqui foi considerado o sistema do berço ao portão (cradle to gate), isto é, as cinzas sendo transportadas até o portão das empresas.

A Figura 1, a seguir, mostra os três cenários de cinzas considerados: o primeiro cenário indica o ciclo de vida básico das cinzas pesadas de carvão mineral desde a extração do carvão mineral até a disposição nas lagoas; o segundo cenário inclui a secagem e o transporte para uso na indústria de materiais cerâmicos; e o terceiro cenário abarca a secagem e o transporte para uso na fabricação de cimentos Portland.

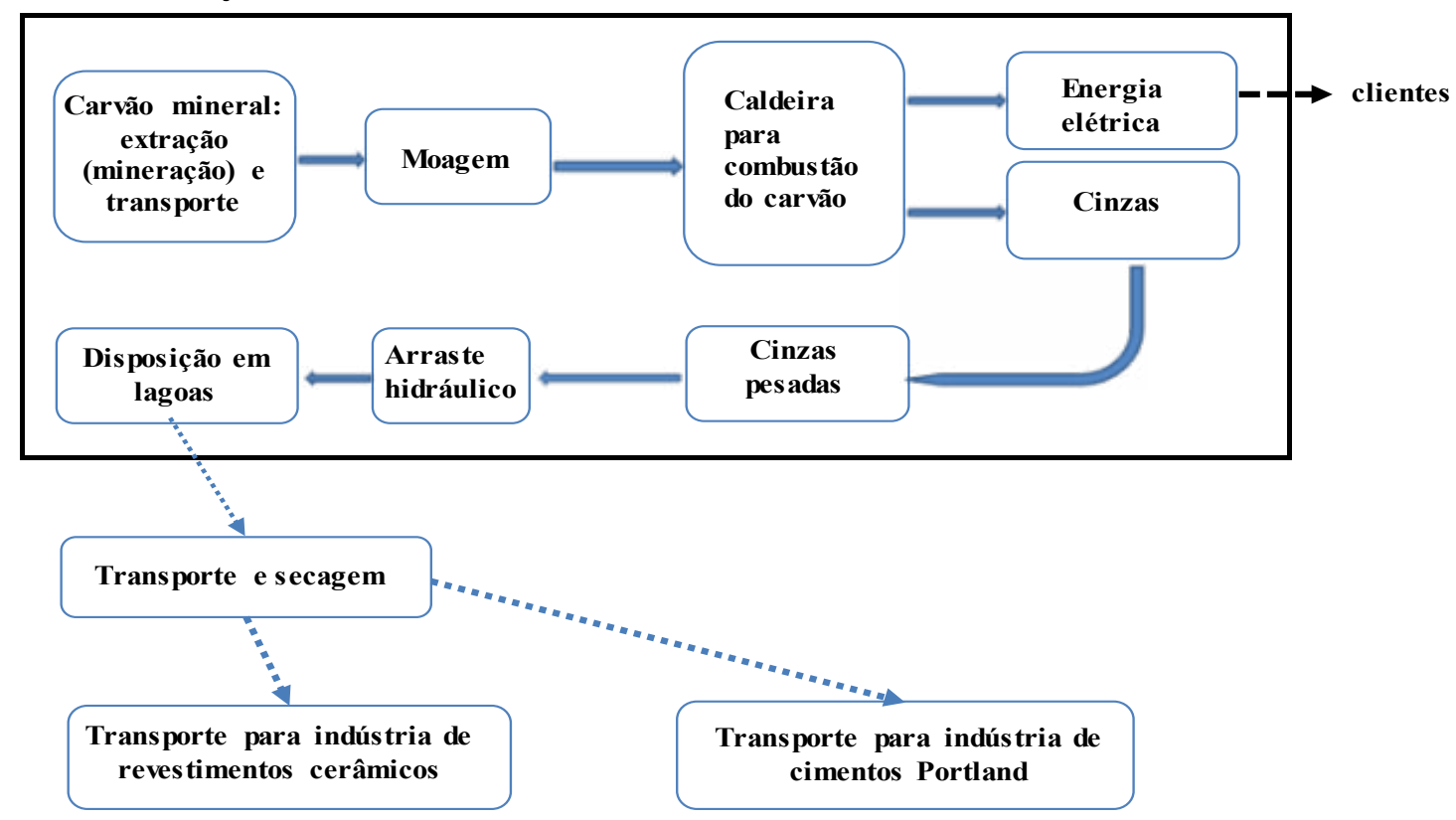

Figura 1 - Limites do sistema de cinzas pesadas Fonte: adaptado de Kniess (2005).

A Figura 2 mostra os limites do processo produtivo da areia desde a extração até o transporte para a indústria de revestimentos cerâmicos. 


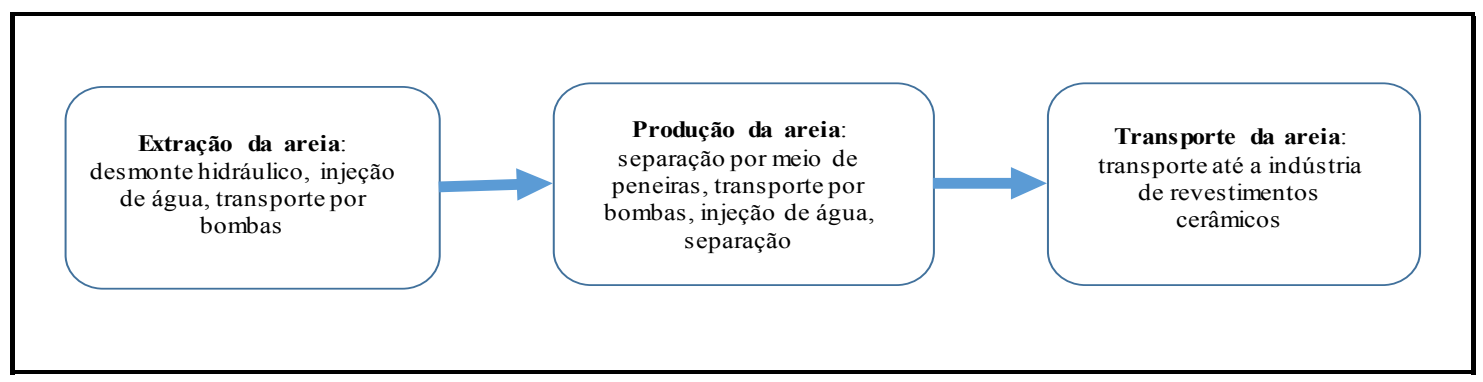

Figura 2 - Limites do sistema produção da areia Fonte: adaptado de Souza (2012).

A Figura 3, a seguir, mostra os limites do processo produtivo do clínquer desde a extração das matérias-primas até a produção do clínquer.

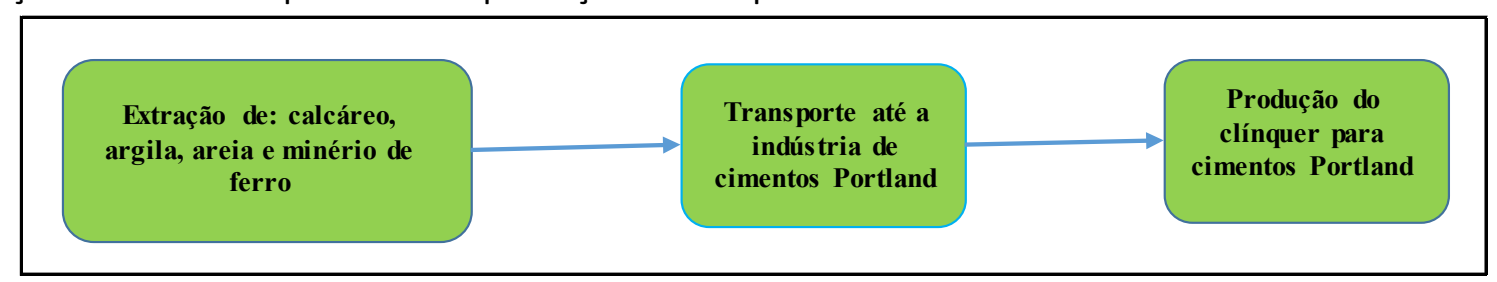

Figura 3 - Limites do sistema de produção do clínquer Fonte: adaptado de Souza (2012).

A unidade funcional utilizada neste estudo foi de $1.000 \mathrm{~kg}$ de cinzas pesadas geradas para as cinzas pesadas; $1.000 \mathrm{~kg}$ de areia produzida para a areia destinada à indústria de revestimentos cerâmicos; e 1.000 kg de clínquer produzido para o clínquer destinado à produção de cimentos Portland. O cálculo do inventário foi baseado em dados primários coletados diretamente na termelétrica e em dados secundários coletados na literatura e na base de dados Ecoinvent do software SimaPro 8.0.

Já para a avaliação dos dados, foram utilizados o software SimaPro e o método ReCiPe endpoint $\mathrm{H}$. Foi escolhido o método ReCiPe porque, além da coerência entre a caracterização no midpoint e a caracterização do midpoint até o endpoint apontada por Hauschild et al. (2013), esse método é normalizado para a Europa (foram utilizados neste estudo valores numéricos europeus) e consta na base de dados do software SimaPro. O método ReCiPe, segundo Goedkoop et al. (2013), avalia os impactos sob três perspectivas diferentes: Individualista (I), uma visão de curto prazo; Hierárquico $(\mathrm{H})$, um modelo de consenso; e Igualitário $(\mathrm{E})$, uma visão de longo prazo. $\mathrm{Na}$ avaliação de impactos do presente estudo, foi escolhida a perspectiva Hierárquico $(H)$, pois, segundo os proponentes do método, este é o modelo de consenso entre os pesquisadores.

\section{RESULTADOS}

Nesta seção, são apresentados os inventários da produção de cinzas pesadas, da produção de cinzas e de areia para uso na indústria de revestimentos e da produção de cinzas e de clínquer para uso na indústria de cimentos Portland. A empresa analisada gerou, em média, para cada 100 toneladas ( $\mathrm{t}$ ) de carvão mineral consumidas, $43 \mathrm{t}$ de cinzas (43\%), das quais $80 \%$ são extraídas secas e $20 \%$ são extraídas úmidas. A umidade das cinzas pesadas é decorrente dos processos de extração e manuseio, sendo, portanto, bastante variável, mas sempre de teor elevado (Kniess, 2005). 


\subsection{Inventários dos processos produtivos}

A Tabela 1, exposta a seguir, apresenta o inventário das cinzas pesadas provenientes da combustão do carvão mineral. O inventário das cinzas dispostas nas lagoas corresponde às colunas (1) e (2); o inventário das cinzas para o uso na indústria de revestimentos cerâmicos consiste nas colunas (1), (2), (3) e (4); e o inventário das cinzas para uso na indústria de cimentos Portland é indicado nas colunas (1), (2), (3) e (5).

Tabela 1 - Ciclo de vida das cinzas pesadas (por $1.000 \mathrm{~kg}$ de cinzas pesadas)

\begin{tabular}{|c|c|c|c|c|c|c|}
\hline \multicolumn{5}{|c|}{ Inventário comum para os dois usos das cinzas } & \begin{tabular}{|l|} 
Uso cerâmica \\
$(4)$
\end{tabular} & \multirow[t]{2}{*}{$\begin{array}{l}\text { Uso cimento } \\
(5)\end{array}$} \\
\hline \multirow[b]{2}{*}{ Aspectos ambientais } & \multirow[b]{2}{*}{ Unidade } & \multicolumn{4}{|c|}{ Valores } & \\
\hline & & $\begin{array}{l}\text { Extração do } \\
\text { carvão (1) }\end{array} \mid$ & $\begin{array}{l}\begin{array}{l}\text { Geração das } \\
\text { cinzas (2) }\end{array} \\
\end{array}$ & \begin{tabular}{llll|} 
Retirada & das & cinzas \\
da & lagoa & e & secagem \\
(3) & & & \\
\end{tabular} & \begin{tabular}{|l|} 
Transporte \\
para indústria \\
de cerâmica
\end{tabular} & $\begin{array}{l}\text { Transporte } \\
\text { para indústria } \\
\text { de cimento } \\
\end{array}$ \\
\hline \multicolumn{7}{|l|}{ Entradas } \\
\hline Carvão ROM & $\mathrm{kg}$ & 28537 & & & & \\
\hline Carvão Energético (CE) & $\mathrm{kg}$ & & 11940,3 & & & \\
\hline Água (lagoa) & $\mathrm{m}^{3}$ & 6,99 & & & & \\
\hline Água (rio) & $\mathrm{m}^{3}$ & & 79,1 & & & \\
\hline Uso do solo & $\mathrm{m}^{2}$ & & 314.000 & & & \\
\hline Diesel & $\mathrm{kg}$ & 1,30 & 3,38 & 0,126 & 0,0825 & 2,06 \\
\hline Eletricidade & $\mathrm{kWh}$ & 46,1 & 156 & 6,50 & & \\
\hline Transporte por trem & $\mathrm{tkm}$ & & 160 & & & \\
\hline Transporte por caminhão & $\mathrm{tkm}$ & & 40 & 2,40 & 4,00 & 100 \\
\hline \multicolumn{7}{|l|}{ Saídas } \\
\hline Carvão energético & $\mathrm{kg}$ & 11940,3 & & & & \\
\hline Cinzas pesadas & $\mathrm{kg}$ & & 1000 & 1000 & 1000 & 1000 \\
\hline \multicolumn{7}{|l|}{ Emissões para o ar } \\
\hline $\mathrm{CH}_{4}$ & $\mathrm{~kg}$ & 2,44 & $1,25 \mathrm{E}-32$ & & & \\
\hline $\mathrm{CO}_{2}$ & $\mathrm{~kg}$ & 2,24 & 1,69 & & & \\
\hline $\mathrm{CO}$ & $\mathrm{kg}$ & & 0,0100 & & & \\
\hline $\mathrm{SO}_{2}$ & $\mathrm{~kg}$ & & 23,2 & & & \\
\hline $\mathrm{N}_{2} \mathrm{O}$ & $\mathrm{kg}$ & & $1,24 \mathrm{E}-04$ & & & \\
\hline $\mathrm{NO}_{2}$ & $\mathrm{~kg}$ & & $1,22 \mathrm{E}-02$ & & & \\
\hline Particulado $<2,5 \mu \mathrm{m}$ & $\mathrm{kg}$ & & 15,4 & & & \\
\hline Particulado $>10 \mu \mathrm{m}$ & $\mathrm{kg}$ & & 3,83 & & & \\
\hline $\begin{array}{l}\text { Particulado }>2,5 \mu \mathrm{m}< \\
10 \mu \mathrm{m}\end{array}$ & $\mathrm{kg}$ & & 1,80 & & & \\
\hline \multicolumn{7}{|l|}{ Emissões para a água } \\
\hline Sólidos totais & $\mathrm{kg}$ & 18,6 & & & & \\
\hline Sulfatos & $\mathrm{kg}$ & 9,55 & & & & \\
\hline Alumínio & $\mathrm{kg}$ & $5,38 \mathrm{E}-02$ & & & & \\
\hline Cobre & $\mathrm{kg}$ & $3,50 \mathrm{E}-05$ & & & & \\
\hline Ferro & $\mathrm{kg}$ & $5,43 \mathrm{E}-03$ & & & & \\
\hline Manganês & $\mathrm{kg}$ & $2,73 \mathrm{E}-02$ & & & & \\
\hline Zinco & $\mathrm{kg}$ & $9,80 \mathrm{E}-04$ & & & & \\
\hline
\end{tabular}

Fonte: adaptado de Restrepo (2012) e Restrepo, Bazzo e Miyake (2015).

Já a Tabela 2 apresenta o inventário do ciclo de vida da areia adaptado por Castro et al. (2015) para as condições brasileiras. 
Tabela 2 - Ciclo de vida da produção de areia (por $1.000 \mathrm{~kg}$ de areia)

\begin{tabular}{|c|c|c|}
\hline Aspectos ambie ntais & Valor & Un i da de \\
\hline \multicolumn{3}{|l|}{ Entradas } \\
\hline Areia & 1000 & kg \\
\hline Diesel & 74,1 & MJ \\
\hline Eletricidade & 0,130 & $\mathbf{k W h}$ \\
\hline Borracha & $2,00 \mathrm{E}-03$ & $\mathrm{~kg}$ \\
\hline Transporte caminhão $3.5-20 \mathrm{t}$ & $3,00 \mathrm{E}-\mathrm{O} 3$ & $\mathbf{t} \mathrm{km}$ \\
\hline Transporte caminhão $20-28 \mathrm{t}$ & $2,92 \mathrm{E}-03$ & t km \\
\hline Transporte caminhão $3.5-20 \mathrm{t}$ para indústria cerâmica & 100 & t km \\
\hline Areia & 1000 & $\mathbf{k g}$ \\
\hline \multicolumn{3}{|l|}{ Resíduos para tratamento } \\
\hline Borracha & $2,00 \mathrm{E}-\mathrm{O} 3$ & kg \\
\hline Óleo lubrificante & 0,0146 & $\mathrm{~kg}$ \\
\hline Aço & 0,0130 & $\mathrm{~kg}$ \\
\hline
\end{tabular}

Fonte: adaptado de Castro et al. (2015).

A Tabela 3, por sua vez, mostra o inventário da produção de clínquer para uma indústria de cimento. Os dados foram adaptados da base de dados Ecoinvent do software SimaPro para a matriz brasileira de água e eletricidade identificados com a sigla (BR) na Tabela a seguir. As demais informações referem-se a dados europeus. 
Tabela 3 - Ciclo de vida do clínquer (por 1.000 kg de clínquer)

\begin{tabular}{|c|c|c|c|c|c|}
\hline Aspectos ambientais & Valor & Unid. & As pectos ambientais & Valor & Unid. \\
\hline Entradas & & & Saídas & & \\
\hline Recursos & & & Produtos & & \\
\hline Água (BR) & 1,62 & $\mathrm{~m}^{3}$ & Clinquer & 1000 & $\mathrm{~kg}$ \\
\hline Materiais/Combus tíveis & & & Emis s ões para o ar & & \\
\hline Amônia & $9,08 \mathrm{E}-01$ & $\mathrm{~kg}$ & Amônia & $2,28 \mathrm{E}-02$ & $\mathrm{~kg}$ \\
\hline Óleo lubrificante & $4,71 \mathrm{E}-02$ & $\mathrm{~kg}$ & Antimônio & $2,00 \mathrm{E}-06$ & $\mathrm{~kg}$ \\
\hline Calcário marga & 466 & $\mathrm{~kg}$ & Arsênico & $1,20 \mathrm{E}-05$ & $\mathrm{~kg}$ \\
\hline Argila & 331 & $\mathrm{~kg}$ & Berílio & $3,00 \mathrm{E}-06$ & $\mathrm{~kg}$ \\
\hline Calcário & 841 & $\mathrm{~kg}$ & Cádmio & $7,00 \mathrm{E}-06$ & $\mathrm{~kg}$ \\
\hline Areia & $9,26 \mathrm{E}+00$ & $\mathrm{~kg}$ & Dióxido de carbono biogênico & 15,1 & $\mathrm{~kg}$ \\
\hline Cal hidratado & $3,92 \mathrm{E}+00$ & $\mathrm{~kg}$ & Dióxido de carbono fóssil & 839 & $\mathrm{~kg}$ \\
\hline Refratário básico & $1,90 \mathrm{E}-01$ & $\mathrm{~kg}$ & Monóxido de carbono, fóssil & $4,72 \mathrm{E}-01$ & $\mathrm{~kg}$ \\
\hline Refratário argila refratária & $8,21 \mathrm{E}-02$ & $\mathrm{~kg}$ & Crômio & $1,45 \mathrm{E}-06$ & $\mathrm{~kg}$ \\
\hline Refratário alto teor de óxido de alumínio & $1,37 \mathrm{E}-01$ & $\mathrm{~kg}$ & Cobalto & $4,00 \mathrm{E}-06$ & $\mathrm{~kg}$ \\
\hline Cimento & $6,27 \mathrm{E}-09$ & $\mathrm{p}$ & Cobre & $1,40 \mathrm{E}-05$ & $\mathrm{~kg}$ \\
\hline Dies el utilizado em máquinas internas & $1,34 \mathrm{E}+01$ & MJ & Dioxina, 2,3,7,8 Tetraclorodibenzo-p- & $9,6 \mathrm{E}-10$ & $\mathrm{~kg}$ \\
\hline Máquinas industriais pesadas & $3,76 \mathrm{E}-02$ & $\mathrm{~kg}$ & Calor perdas & 3620 & MJ \\
\hline Eletricidade (BR) & 58,0 & $\mathrm{kWh}$ & Cloreto de hidrogênio & $6,31 \mathrm{E}-03$ & $\mathrm{~kg}$ \\
\hline Carvão mineral & 35,4 & $\mathrm{~kg}$ & Chumbo & $8,50 \mathrm{E}-05$ & $\mathrm{~kg}$ \\
\hline Bauxita & $1,20 \mathrm{E}-01$ & $\mathrm{~kg}$ & Mercúrio & $3,30 \mathrm{E}-05$ & $\mathrm{~kg}$ \\
\hline Aço cromo $18 / 8$ & $5,86 \mathrm{E}-02$ & $\mathrm{~kg}$ & Metano fóssil & $8,88 \mathrm{E}-03$ & $\mathrm{~kg}$ \\
\hline Gás natural, high pressure & 6,81 & MJ & Níquel & $5,00 \mathrm{E}-06$ & $\mathrm{~kg}$ \\
\hline Óleo combustível pesado & 25,5 & $\mathrm{~kg}$ & Óxidos de nitrogênio & 1,08 & $\mathrm{~kg}$ \\
\hline Óleo combustível leve & $3,74 \mathrm{E}-01$ & $\mathrm{~kg}$ & $\begin{array}{l}\text { NMVOC compostos orgânicos voláteis } \\
\text { não metânicos }\end{array}$ & $5,64 \mathrm{E}-02$ & $\mathrm{~kg}$ \\
\hline Coque de petróleo & 3,91 & $\mathrm{~kg}$ & Particulados $<2.5 \mathrm{um}$ & 2,41E-02 & $\mathrm{kg}$ \\
\hline Transporte caminhão $3.5-20 t$ & $8,61 \mathrm{E}-02$ & $\mathrm{tkm}$ & Particulados $>10 \mathrm{um}$ & $5,66 \mathrm{E}-03$ & $\mathrm{~kg}$ \\
\hline Transporte caminhão $20-28 t$ & 2,68 & $\mathrm{tkm}$ & Particulados $>2.5$ um e $<10$ um & 7,92E-03 & $\mathrm{kg}$ \\
\hline Transporte, caminhão >28t & 2,11 & $\mathrm{tkm}$ & Selênio & $2,00 \mathrm{E}-06$ & $\mathrm{~kg}$ \\
\hline Transporte Furgão $<3.5 \mathrm{t}$ & $7,09 \mathrm{E}-02$ & $\mathrm{tkm}$ & Dióxido de enxofre & $3,55 \mathrm{E}-01$ & $\mathrm{~kg}$ \\
\hline Transporte barcaça & 7,22 & $\mathrm{tkm}$ & Tálio & $1,30 \mathrm{E}-05$ & $\mathrm{~kg}$ \\
\hline Transporte ferroviário & 24,8 & $\mathrm{tkm}$ & Estanho & $9,00 \mathrm{E}-06$ & $\mathrm{~kg}$ \\
\hline \multirow[t]{6}{*}{ Água tratada (consumidor) } & 340 & $\mathrm{~kg}$ & Vanádio & $5,00 \mathrm{E}-06$ & $\mathrm{~kg}$ \\
\hline & & & Zinco & $6,00 \mathrm{E}-05$ & $\mathrm{~kg}$ \\
\hline & & & Cromo VI & $5,5 \mathrm{E}-07$ & $\mathrm{~kg}$ \\
\hline & & & Resíduos para tratamento & & \\
\hline & & & $\begin{array}{l}\text { Disposição, resíduo inerte, } 5 \% \text { de } \\
\text { água, aterro para material inerte. }\end{array}$ & $8,00 \mathrm{E}-02$ & $\mathrm{~kg}$ \\
\hline & & & $\begin{array}{l}\text { Disposição, resíduo sólido urbano, } \\
22.9 \% \text { de água, para incineracão }\end{array}$ & $4,50 \mathrm{E}-02$ & $\mathrm{~kg}$ \\
\hline
\end{tabular}

Fonte: adaptado do SimaPro (2015). 


\subsection{Impactos ambientais medidos pela pontuação única}

A Tabela 4, a seguir, apresenta os dados de impactos ambientais calculados pelo software SimaPro, baseados nos inventários das Tabelas 1, 2 e 3, utilizando o método ReCiPe endpoint H pontuação única. Já a Figura 4 mostra de forma gráfica as informações presentes na Tabela 4.

Tabela 4 - Impactos ambientais calculados pelo método ReCiPe endpoint $\mathrm{H}$ pontuação única

\begin{tabular}{l|c|c|c|c}
\hline \multicolumn{1}{c|}{ Proces so } & \multicolumn{3}{c}{ Pontuação } \\
\cline { 2 - 5 } & Sáude humana & Ecos sis tema & Recurs os & Total \\
\hline Geração de cinzas pela termelétrica & 163,6 & 32,6 & 0,21 & 196,4 \\
Geração de cinzas para cerâmica & 163,7 & 32,6 & 0,21 & 196,5 \\
Geração de cinzas para cimento & 164,9 & 33,2 & 0,212 & 198,3 \\
Produção de clínquer & 28,2 & 16,2 & 0,084 & 44,5 \\
Produção de areia & 1,6 & 0,7 & 0,001 & 2,3 \\
Total & 522 & 115,3 & 0,72 & 638,1 \\
\hline
\end{tabular}

Fonte: elaborada a partir dos dados do SimaPro (2015).

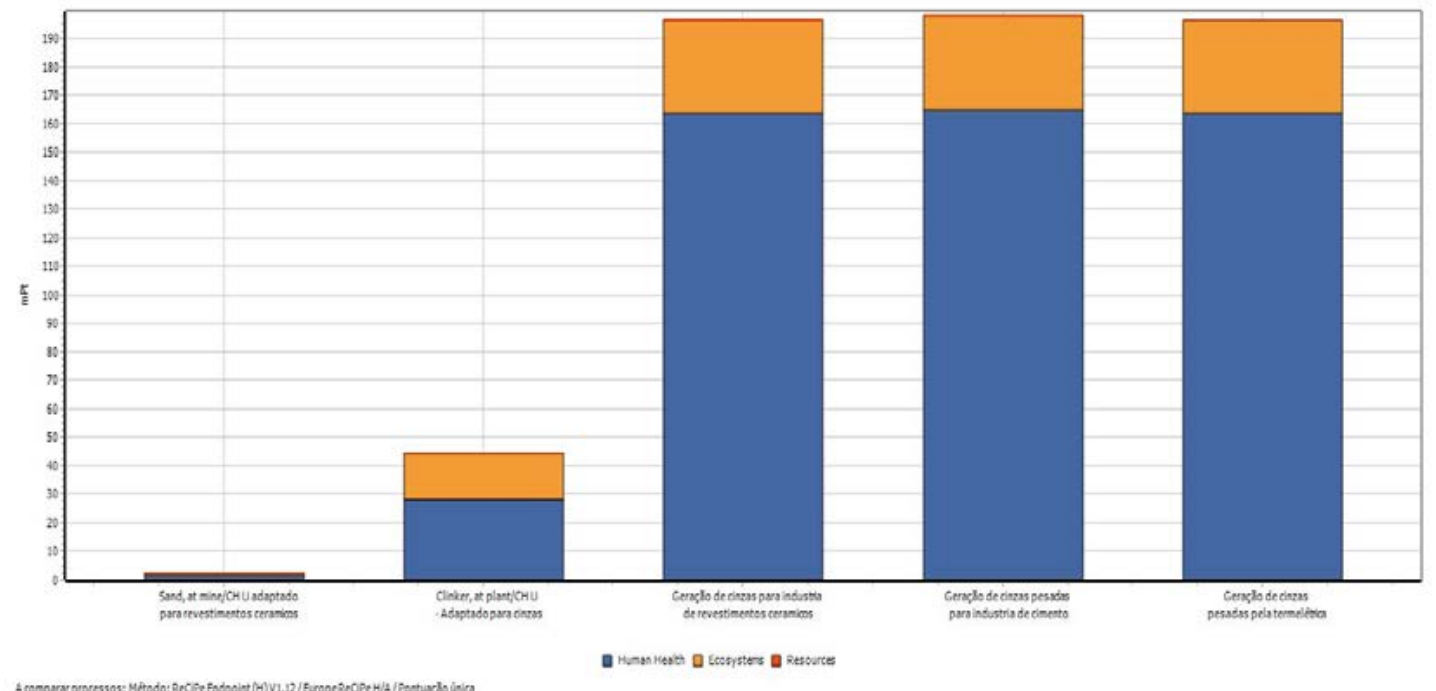

Figura 4 - Comparação entre os processos por meio do método ReCiPe endpoint H pontuação única Fonte: elaborada a partir dos dados do SimaPro (2015).

Com base nesses dados, observa-se que a categoria Saúde humana (Human Health) é a mais afetada pelos cinco cenários: a soma dos valores totais dos pontos de impacto $(198,3+$ $196,6+196,4+44,5+2,3)$ totaliza 638,1 pontos, dos quais 522 pertencem à Saúde humana, o que representa $81,8 \%$ da pontuação única total. A categoria Ecossistema (Ecosystems), por sua vez, totaliza 115,3 pontos, representando $18,1 \%$ da pontuação única total, enquanto que a categoria Recursos (Resources) representa somente $0,1 \%$ dos impactos avaliados.

\subsection{Cálculo da ecoeficiência conforme a norma NBR ISO 14045}

O valor do produto adotado foi a geração diária (em toneladas) de cinzas pesadas pela termelétrica, considerando-se a média diária no ano de 2010, isto é, 804,65 t. Foi utilizado o valor referente ao ano de 2010 porque os dados aqui utilizados são referentes às pesquisas de Restrepo (2012) e Restrepo, Bazzo e Miyake (2015) realizada nesse mesmo ano na usina em questão. 
O impacto ambiental utilizado na medida de ecoeficiência foi calculado por meio do software SimaPro utilizando o método ReCiPe endpoint $\mathrm{H}$ normalizado conforme apresentado na Tabela 4. De acordo com a Equação 1, os resultados são:

I. Ecoeficiência da termelétrica na geração das cinzas pesadas $=804,65 / 196,4=4,10$

II. Ecoeficiência da termelétrica no uso das cinzas para revestimentos cerâmicos = $804,65 / 196,5=4,09$

III. Ecoeficiência da termelétrica no uso das cinzas na indústria de cimento = $804,65 / 198,3=4,06$

Comparando-se, por meio da Equação 2, a ecoeficiência dos processos de geração de cinzas para uso na indústria de revestimentos cerâmicos com a ecoeficiência da termelétrica na geração de cinzas pesadas, que é o produto base deste estudo, tem-se o Fator1 =4,09/4,10 =0,998.

Esse resultado mostra que o uso das cinzas pesadas não aumentaria a ecoeficiência da termelétrica, pois, para uso na indústria de revestimentos cerâmicos, a ecoeficiência ainda ficaria em 0,998 (99,8\%), ou seja, aproximadamente $0,2 \%$ inferior em relação à disposição das cinzas nas lagoas.

As cinzas pesadas poderiam substituir toda a areia na produção de revestimentos cerâmicos, o que cessaria os impactos ambientais da produção de areia mencionados na Tabela 4. Desse modo, o valor que representa esse impacto (2,3 pontos) pode ser retirado para efeito de cálculo dos impactos da geração das cinzas pesadas pela termelétrica, pois, pela metodologia ACV, impactos evitados têm o valor negativo. Refazendo-se os cálculos para esse caso da produção de areia, a ecoeficiência da termelétrica com o uso das cinzas para revestimentos cerâmicos é dada por 804,65/(196,6 - 2,3) = 4,14.

Comparando esse novo resultado com a ecoeficiência da termelétrica na geração de cinzas pesadas, tem-se o Fator1a $=4,14 / 4,10=1,0097$

Isso indica que a ecoeficiência da termelétrica passaria de 0,998 para 1,0097, ou seja, passaria de $0,2 \%$ inferior para $0,97 \%$ superior. São valores percentualmente pequenos, mas que alteram o valor da ecoeficiência da empresa para determinado produto, visto que, por enquanto, as cinzas são sempre geradas independentemente da avaliação ambiental ou do processo de gestão da produção.

Comparando a geração de cinzas pesadas para uso na indústria de cimentos Portland com a geração de cinzas pela termelétrica, obtém-se o Fator2 $=4,06 / 4,10=0,990$.

Esse fator mostra que o uso das cinzas na substituição do clínquer na indústria de cimento não aumentaria a ecoeficiência da termelétrica, pois a ecoeficiência ficaria em 0,99, isto é, aproximadamente $1 \%$ inferior em relação à disposição das cinzas nas lagoas.

Como se sabe, as cinzas pesadas não podem substituir totalmente o uso do clínquer na produção de cimento. A norma ABNT NBR 5736: 1991 limita o uso de material pozolânico na constituição do cimento Portland CP IV a 15-50\% em massa (ABNT, 1991). Por esse motivo, não foi possível utilizar o mesmo procedimento do Fator $_{1}$, diminuindo o valor total da pontuação referente ao processo produtivo do clínquer.

Supondo que a substituição progressiva do clínquer na produção de cimento Portland pelas cinzas pesadas, conforme os limites estabelecidos pela norma supracitada, tenha como consequência a redução proporcional do impacto ambiental medido pela pontuação única mostrada na Tabela 4 e aplicando as equações 1 e 2, obtêm-se os valores apresentados na Tabela 5. Como é possível perceber, a substituição progressiva do uso do clínquer por cinzas pesadas na produção de cimento Portland melhora a ecoeficiência da termelétrica já a partir de $15 \%$. Quando se substitui 
$50 \%$ do clínquer por cinzas pesadas na indústria de cimento Portland, a ecoeficiência da termelétrica pode chegar a 11,5\% superior em comparação à disposição final das cinzas nas lagoas.

Tabela 5 - Substituição parcial do clínquer por cinzas pesadas

\begin{tabular}{|c|c|c|c|c|c|}
\hline $\begin{array}{l}\text { Substituição } \\
\text { do clínquer } \\
(\%)\end{array}$ & $\begin{array}{l}\text { Redução do impacto do } \\
\text { clínquer (pontuação) }\end{array}$ & 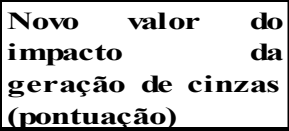 & $\begin{array}{l}\text { Ecoeficiência } \\
\text { geração cinzas }\end{array}$ & Fator2a & Porcentagem \\
\hline 15 & 6,68 & 191,62 & 4,20 & 1,024 & 2,4 \\
\hline 20 & 8,90 & 189,40 & 4,25 & 1,037 & 3,7 \\
\hline 25 & 11,13 & 187,17 & 4,30 & 1,049 & 4,9 \\
\hline 30 & 13,35 & 184,95 & 4,35 & 1,061 & 6,1 \\
\hline 35 & 15,58 & 182,72 & 4,40 & 1,073 & 7,3 \\
\hline 40 & 17,80 & 180,50 & 4,46 & 1,088 & 8,8 \\
\hline 45 & 20,03 & 178,27 & 4,51 & 1,100 & 10,0 \\
\hline 50 & 22,25 & 176,05 & 4,57 & 1,115 & 11,5 \\
\hline
\end{tabular}

Fonte: elaborada pelos autores.

\section{ANÁLISE E DISCUSSÃO DOS RESULTADOS}

Verificando-se os valores comparativos calculados de ecoeficiência da termelétrica nos dois processos, o uso na indústria de revestimentos cerâmicos tem a possibilidade de aumentar a ecoeficiência de 0,998 (ou menos $0,2 \%$ ) para 1,0097 (ou mais $0,97 \%$ ), e o uso na indústria de cimento Portland tem a possibilidade de aumentar esse índice de 0,990 (ou menos 1\%) para 1,024 a 1,115 (ou mais $2,4 \%$ a $11,5 \%$ ). Assim, pode-se inferir que o uso das cinzas pesadas na substituição do clínquer na produção de cimento Portland é a opção ambiental mais vantajosa para a termelétrica. Isso indica que o uso das cinzas pesadas, um resíduo inerente ao processo de combustão de carvão, em substituição à areia ou ao clínquer, melhora a ecoeficiência das empresas termelétricas.

Além, disso, a substituição do clínquer diminui os impactos ambientais na produção de cimento Portland. Algumas pesquisas mostram que a substituição do clínquer por resíduos industriais diminui os impactos ambientais tanto do cimento quanto do concreto. Gäbel e Tillman (2005) citam que uma opção interessante na produção de cimento consiste no aumento da utilização de subprodutos industriais e resíduos como aditivos, matérias-primas ou combustível. Um aumento na utilização de subprodutos e resíduos substitui o uso de recursos minerais naturais e reduz o consumo total das matérias-primas. $O$ aumento da utilização desses materiais em substituição às matérias-primas originais tem como consequência a redução das emissões de dióxido de carbono $\left(\mathrm{CO}_{2}\right)$, de monóxido de carbono (CO) e de compostos orgânicos voláteis (VOC). Reduções nas emissões de óxidos de nitrogênio $\left(\mathrm{NO}_{x}\right)$ e dióxido de enxofre $\left(\mathrm{SO}_{2}\right)$ são observadas com o uso de subprodutos e resíduos na mistura final para a fabricação do cimento.

Chen et al. (2010) afirmam que, para reduzir o impacto ambiental do cimento e do concreto, as indústrias têm sido estimuladas, ao longo dos últimos dez anos, a aumentar a substituição de cimento Portland por materiais alternativos que consistem, principalmente, em resíduos ou subprodutos industriais. Nesse sentido, estudos têm confirmado que o cimento Portland é a principal fonte de $\mathrm{CO}_{2}$ gerado por misturas de concreto típicas produzidas comercialmente, sendo responsável por $74-81 \%$ das emissões de $\mathrm{CO}_{2}$ do concreto. 


\section{CONSIDERAÇÕES FINAIS}

Este artigo apresentou um método para mensurar a ecoeficiência, a fim de avaliar alternativas de valorização de resíduos gerados em processos produtivos. Esse método foi aplicado a uma situação concreta: as cinzas pesadas geradas pela queima de carvão mineral de uma termelética.

A utilização de resíduos gerados em processos de produção como matérias-primas em outros processos tem sido apontada como uma solução para os problemas ambientais a ser utilizada pelas empresas. Para o meio ambiente, essa forma de valorização de resíduos contribui de duas maneiras: de um lado, diminui a necessidade de extração de recursos naturais e, de outro, diminui o lançamento de poluentes, o que melhora a qualidade do meio ambiente. Como visto, a valorização da cinza pesada, além de eliminar a sua acumulação em lagoas, reduz as emissões de $\mathrm{CO}_{2}$ quando utilizada para substituir o clínquer na fabricação de cimento. Para a empresa geradora do resíduo, reduz os custos das atividades de controle da poluição pela redução da necessidade de efetuar a disposição final de resíduos de modo seguro. Além disso, gera receitas, como é o caso das cinzas pesadas estudadas. Para a empresa usuária do resíduo, os benefícios não são menores, começando pelo fato de que os resíduos em geral custam menos que as matérias-primas virgens.

Como os benefícios potenciais da valorização de resíduos variam entre alternativas tecnicamente disponíveis, a aplicação de métodos apropriados de avaliação torna-se necessária. A avaliação baseada na mensuração da ecoeficiência apresenta-se de início coerente, pois ecoeficiência refere-se à combinação de questões ambientais e econômicas. Porém, essa coerência se perde se a mensuração não considerar o processo produtivo ampliado, ou seja, o ciclo de vida do produto, motivo pelo qual a NBR ISO 14045 recomenda que os impactos ambientais sejam avaliados com a utilização da ACV.

Dentro do limite do presente estudo, pode-se inferir que a medida de ecoeficiência ambiental deve ser realizada da seguinte forma: avaliar os impactos ambientais considerando os aspectos ambientais envolvidos no processo produtivo desde a extração das matérias-primas até a disposição final do(s) resíduo(s) e comparar tais impactos com o processo produtivo em que há a inclusão do uso desses resíduos; utilizar, para isso, a ACV do resíduo (ou dos resíduos) a ser valorizado; verificar quais são os impactos ambientais e se há possiblidade de minimizá-los ou eliminá-los; e calcular o valor da ecoeficiência do processo como recomenda a NBR ISO 14045.

Uma limitação deste método se refere aos dados para a elaboração do inventário, que nem sempre estão disponíveis. No exemplo apresentado, parte dos dados foram retirados da literatura e de bases de dados residentes no software SimaPro referentes a empresas europeias e que podem não refletir as condições encontradas nas empresas brasileiras. Ademais, as emissões para o ar, o solo e a água e as condições de tratamento de resíduos também concernem a países europeus e podem ter especificidades diferentes das condições brasileiras. Essa é uma questão que pode ser resolvida no âmbito de uma empresa, pois requer um esforço de dimensão nacional com amplo apoio de intituições de pesquisa. Outra limitação diz respeito ao limite do sistema para efeito da ACV. Devido à disponibilidade de dados e de tempo, os estudos sobre ACV dificilmente conseguem alcançar todos os estágios do ciclo de vida, do berço ao túmulo (cradle to grave), conforme recomenda a literatura e a norma citada, de modo que, em geral, os limites incluem apenas os estágios sob o controle da empresa e alguns realizados por fornecedores de primeira camada. Apesar dessas limitações, o uso da ecoeficiência calculada a partir da ACV, como mostrado neste artigo, pode indicar a melhor solução para valorizar os resíduos inerentes de um processo produtivo. 


\section{REFERÊNCIAS}

Asokan, P.; Saxena, M.; Asolekar, S. R. Coal combustion residues - environmental implications and recycling potentials. Resources, Conservation and Recycling, v. 43, n. 3, p. 239-262, 2005.

Associação Brasileira de Normas Técnicas (ABNT). NBR 5736: Cimento Portland pozolânico. Rio de Janeiro, 1991.

Associação Brasileira de Normas Técnicas (ABNT). NBR ISO 14040: Gestão ambiental - avaliação do ciclo de vida - princípios e estrutura. Rio de Janeiro, 2009a.

Associação Brasileira de Normas Técnicas (ABNT). NBR ISO 14044: Gestão ambiental - avaliação do ciclo de vida - requisitos e orientações. Rio de Janeiro, 2009b.

Associação Brasileira de Normas Técnicas (ABNT). NBR ISO 14045: Gestão ambiental - avaliação da ecoeficiência de sistema de produto - princípios, requisitos e orientações. Rio de Janeiro, 2014.

ASTRUP, T. F. et al. Life cycle assessment of thermal waste-to-energy technologies: Review and recommendations. Waste Management, v. 37, p. 104-115, 2015.

Babbitt, C. W.; Lindner, A. S. A life cycle inventory of coal used for electricity production in Florida. Journal of Cleaner Production, v. 13, n. 9, p. 903-912, 2005.

Barbieri, J. C. Gestão ambiental empresarial: conceitos, modelos e instrumentos. São Paulo: Saraiva, 2007.

Bautista-Lazo, S.; Short, T. Introducing the all seeing eye of business: a model for understanding the nature, impact and potential uses of waste. Journal of Cleaner Production, v. 40, p. 141-150, 2013.

Bréchet, T.; Li, S. The many traps of green technology promotion. Environmental Economics and Policy Studies, v. 15, n. 1, p. 73-91, 2013.

Campos, L. F. A. et al. Planejamento experimental no estudo da maximização do teor de resíduos em blocos e revestimentos cerâmicos. Cerâmica, v. 53, n. 328, p. 373-380, 2007.

Castro, A. L. et al. Análise da viabilidade técnica da adaptação de dos internacionais de inventário de ciclo de vida para o contexto brasileiro: um estudo de caso do concreto para paredes moldadas no local. In: Congresso Brasileiro do Concreto. 57., 2015, Bonito. Anais... Bonito, 2015.

Chen, C. et al. LCA allocation procedure used as an initiative method for waste recycling: an application to mineral additions in concrete. Resources, Conservation and Recycling, v. 54, n. 12, p. 1231-1240, 2010.

Chowdhury, R.; Apul, D.; Fry, T. A life cycle based environmental impacts assessment of construction materials used in road construction. Resources, Conservation and Recycling, v. 54, n. 4, p. 250-255, 2010.

Ekvall, T. et al. What life-cycle assessment does and does not do in assessments of waste management. Waste Management, v. 27, n. 8, p. 989-996, 2007.

Fungaro, D. A.; Izidoro, J. C. Remediação de drenagem ácida de mina usando zeólitas sintetizadas a partir de cinzas leves de carvão. Revista Química Nova, v. 29, n. 4, p. 735-740, 2006.

Gäbel, K.; Tillman, A. M. Simulating operational alternatives for future cement production. Journal of Cleaner Production, v. 13, n. 13-14, p. 1246-1257, 2005.

GLAVIC, P.; LUKMAN, R. Review of sustainability terms and their definitions. Journal of Cleaner Production, v. 15, n. 18, p. 1875-1885, 2007. 
Goedkoop, M. et al. ReCiPe 2008: A life cycle impact assessment method, which comprises harmonised category indicators at the midpoint and the endpoint level. v. 1.08, p.1126, 2013. Disponível em: <http://www.presustainability.com/recipe>. Acesso em 20 maio 2015.

Hauschild, M. S. et al. Identifying best existing practice for characterization modeling in life cycle impact assessment. International Journal of Life Cycle Assessment, v. 18, n. 3, p. 683-697, 2013.

Hodge, M.; Ochsendorf, J.; Fernández, J. Quantifying potential profit from material recycling: a case study in brick manufacturing. Journal of Cleaner Production, v. 18, n. 12, p. 1190-1199, 2010.

International Standard - ISO 14045. Environmental management - Eco-efficiency assessment of product systems - Principles, requirements and guidelines. Geneva: Switzerland, 2012.

Kniess, C. T. Desenvolvimento e caracterização de materiais cerâmicos com adição de cinzas pesadas de carvão mineral. 2005. $253 \mathrm{f}$. Tese (Doutorado em Ciência e Engenharia de Materiais) - Universidade Federal de Santa Catarina, Florianópolis, 2005.

Kniess, C. T. et al. Obtenção e caracterização de produtos cerâmicos com a adição de cinzas pesadas de carvão mineral provenientes de usinas termelétricas. In: Congresso Brasileiro de Cerâmica, 55., 2011, Porto de Galinhas, Anais... Porto Alegre, 2011.

Knight, P.; Jenkins, J. O. Adopting and applying eco-design techniques: a practitioner's perspective. Journal of Cleaner Production, v. 17, n. 5, p. 549-558, 2009.

LAURENT, A. et al. Review of LCA studies of solid waste management systems - Part I: Lessons learned and perspectives. Waste Management, v. 34, n. 3, p. 573-588, 2014a.
LAURENT, A. et al. Review of LCA studies of solid waste management systems - Part II: Methodological guidance for a better practice. Waste Management, v. 34, n. 3, p. 589-606, 2014b.

Liang, S.; Zhang, T. Comparing urban solid waste recycling from the viewpoint of urban metabolism based on physical input-output model: a case of Suzhou in China. Waste Management, v. 32, n. 1, p. 220-225, 2012.

Menéndez, E. et al. Characterization of bottom ashes from coal pulverized power plants to determine their potential use feasibility. Boletin de la Sociedad Española de Cerámica y Vidrio, v. 52, n. 6, p. 296-304, 2013.

Merrild, H.; Larsen, A. W.; Christensen, T. H. Assessing recycling versus incineration of key materials in municipal waste: the importance of efficient energy recovery and transport distances. Waste Management, v. 32, n. 5, p. 1009-1018, 2012.

PILAR, R. et al. Avaliação de pastas de cimento Portland contendo cinza pesada moída. Revista Matéria, v. 21, n. 01, p. 92-104, 2016.

Ramadoss, P.; Sundararajan, T. Utilization of lignite-based bottom ash as partial replacement of fine aggregate in masonry mortar. Arabian Journal for Science and Engineering, v. 39, n. 2, p. 737-745, 2014.

Restrepo, A. H. V. Metodologia de análise e avaliação exergoambiental de plantas termoelétricas operadas em combustão combinada carvão-biomassa. 2012. $177 \mathrm{f}$. Tese (Doutorado em Engenharia Mecânica) - Universidade Federal de Santa Catarina, Florianópolis, 2012.

Restrepo, A. H. V.; Bazzo, E.; Miyake, R. A life cycle assessment of the Brazilian coal used for electric power generation. Journal of Cleaner Production, v. 92, p. 179-186, 2015.

Ruiz, M. S. et al. Abordagens de conflitos socioambientais em casos de subsidência de 
minas de carvão no Brasil e EUA. Ambiente \& Sociedade, v. 17, n. 2, p. 129-156, 2014.

SANTOS, M. R.; TEIXEIRA, C. E.; KNIESS, C. T. Avaliação de desempenho ambiental na valorização de resíduos sólidos de processos industriais. Revista de Administração da UFSM, v. 7, Edição Especial, p. 75-92, 2014.

SimaPro Database. Pré Consultants, Amersfoot, Netherlands, 2015.

Sindicato Nacional da Indústria do Cimento (SNIC). Processo de produção. Disponível em <http://www.snic.org.br/processo.asp>. Acesso em: 11 maio 2015.

Souza, M. P. R. Avaliações das emissões de $\mathrm{CO} 2$ antrópico associadas ao processo de produção do concreto, durante a construção de um edifício comercial, na Região Metropolitana de São Paulo. 2012. 127 f. Dissertação (Mestrado em Tecnologia Ambiental), Instituto de Pesquisas Tecnológicas do Estado de São Paulo, São Paulo, 2012.

Teixeira, C. E. Évolution biochimique des résidus de désencrage dans un contexte de valorisation comme barrière de recouvrement. 2001. Tese (Doutorado em Engenharia Civil e Ambiental) - Université de Sherbrooke, Québec, 2001.

TIAN, J.; CHEN, M. Sustainable design for automotive products: dismantling and recycling

of end-of-life vehicles. Waste Management, $v$. 34, n. 2, p. 458-467, 2014.

Tonini, D.; Astrup, T. Life-cycle assessment of a waste refinery process for enzymatic treatment of municipal solid waste. Waste Management, v. 32, n. 1, p. 165-176, 2012.

VAN Oss, H. G.; Padovani, A. C. Cement manufacture and the environment - part II: environmental challenges and opportunities. Journal of Industrial Ecology, v. 7, n. 1, p. 93126, 2003.
Zanchet, A. et al. Propriedades reométricas e mecânicas e morfologia de compósitos desenvolvidos com resíduos elastoméricos vulcanizados. Polímeros: Ciência e Tecnologia, v. 17, n. 1, p. 23-27, 2007. 